\title{
Effective conductivity of a composite material with stiff imperfect contact conditions 1
}

\author{
L.P. Castro*, D. Kapanadze ${ }^{\sharp}$, E. Pesetskaya $\sharp^{\sharp}$ \\ * CIDMA \& Department of Mathematics, University of Aveiro, Portugal \\ $\sharp$ A. Razmadze Mathematical Institute, Tbilisi State University, Georgia
}

\begin{abstract}
We present an analytic and numerical analysis of several properties of a composite material with stiff imperfect interface conditions. Spaces of functions are identified where we can guarantee existence and uniqueness of solutions. In particular, formulas for the temperature distribution and flux are exhibited. Numerical calculations of the material characteristics such as temperature, flux and the effective conductivity are also performed and interpreted.
\end{abstract}

Keywords: unbounded 2D doubly periodic composite material, effective conductivity, stiff imperfect contact, functional equations

\section{Introduction}

This work is concerned with the analysis of composite material properties within certain 2D structures. Here, the theory of analytical and harmonic functions plays a fundamental role in the mathematical understanding of the problems. In particular, in most cases, by using such theories, it is already possible to describe in explicit form some characteristics of those $2 \mathrm{D}$ structures for different configurations (see [1, 2, 3, 4, 5], etc.). Typically, the obtained formulas depend on several basic parameters of the models. One of the most significant advantages of such approach is that it allows a natural analysis of the behavior of the models when the parameters change. In addition, in agreement with homogenization theory (cf. [6]), fibrous composite

\footnotetext{
${ }^{1}$ This is the accepted version of the following article: L.P. Castro, D. Kapanadze, E. Pesetskaya, Effective conductivity of a composite material with stiff imperfect contact conditions, Mathematical Methods in the Applied Sciences 38(18) (2015), 4638-4649, which has been published in final form at http://onlinelibrary.wiley.com/doi/10.1002/mma.3423/pdf. This article may be used for non-commercial purposes in accordance with the Wiley Self-Archiving Policy http://olabout.wiley.com/WileyCDA/Section/id-820227.html.

${ }^{2}$ Corresponding author.
} 
materials with a large number of cylindrical parallel inclusions can be geometrically represented as a 2D doubly periodic model with disjoint inclusions. Moreover, their properties can be studied on the basis of one representative cell which contains all material features.

From the mathematical point of view, a 2D model with disjoint inclusions is just a multiply connected domain. From the physical perspective, the fields in the composite components (the matrix and inclusions) are supposed to be potentials. This means that they satisfy the Laplace equation in each inner point of the considered domain. In addition, it is well-known that for determining the properties of composite bodies the interfaces between solids play a significant role. This is considered in the form of boundary conditions to be defined upon the mechanical assumptions. In the existing literature, the most usual interfaces are treated as ideal contact interface, or as soft imperfect interface (see [1, 2, 3, 4, 5, 7, 8, 9, 10, 11, and the references therein).

In the case of stiff imperfect interfaces, some properties of composites with randomly distributed components were studied by means of asymptotic and numeric procedures by several authors (cf. [6, 12, 13, 14, etc.). An accurate asymptotic technique to the investigation of an elastic field near the tip of the interface crack lying at soft and stiff imperfect interfaces is applied in [15]. An asymptotic analysis and also FEM-evaluation of imperfect transmission conditions has been performed for a modelling problem of an elastic structure with a thin intermediate interface by [16]. Classification of imperfect soft and stiff interfaces in two-dimensional elasticity was done in [14. There, it was shown that, depending on the stiffness of a thin curved isotropic layer with respect to the neighboring media, there exist four distinct regimes of stiff imperfect interface conditions. The effective behavior of piezoelectric and piezomagnetic circular fibrous composites with stiff imperfect interfaces under longitudinal shear with in-plane electromagnetic fields was recently studied in [17], generalizing the classic work of Rayleigh in a periodic conductive perfect composite.

In this paper, a steady-state heat conduction problem in $2 \mathrm{D}$ unbounded doubly periodic composite materials with stiff imperfect interface conditions is considered. By introducing complex potentials, the corresponding boundary value problem for the Laplace equation is transformed into a special $R$ linear boundary value problem for doubly periodic analytic functions. The method of functional equations is used for obtaining an analytic solution. It allows to compute the average property and reconstruct the temperature and 
the flux at an arbitrary point of such composite model.

\section{Formulation of the problem}

We now turn to the precise formulation of the problem under study. We consider a lattice defined by the two fundamental translation vectors " 1 " and " $\imath$ " (where $\imath^{2}=-1$ ) in the complex plane $\mathbb{C} \cong \mathbb{R}^{2}$ (with the standard notation $z=x+\imath y)$. The representative cell is the unit square

$$
Q_{(0,0)}:=\left\{z=t_{1}+\imath t_{2} \in \mathbb{C}:-\frac{1}{2}<t_{p}<\frac{1}{2}, p=1,2\right\} .
$$

Let $\mathcal{E}:=\bigcup_{m_{1}, m_{2}}\left\{m_{1}+\imath m_{2}\right\}$ be the set of the lattice points, where $m_{1}, m_{2} \in \mathbb{Z}$. The cells corresponding to the points of the lattice $\mathcal{E}$ will be denoted by

$$
Q_{\left(m_{1}, m_{2}\right)}=Q_{(0,0)}+m_{1}+\imath m_{2}:=\left\{z \in \mathbb{C}: z-m_{1}-\imath m_{2} \in Q_{(0,0)}\right\} .
$$

It is considered the situation when mutually disjoint disks (inclusions) of different radii $D_{k}:=\left\{z \in \mathbb{C}:\left|z-a_{k}\right|<r_{k}\right\}$ with the boundaries $\partial D_{k}:=$ $\left\{z \in \mathbb{C}:\left|z-a_{k}\right|=r_{k}\right\}(k=1,2, \ldots, N)$ are located inside the cell $Q_{(0,0)}$ and periodically repeated in all cells $Q_{\left(m_{1}, m_{2}\right)}$. Let us denote by

$$
D_{0}:=Q_{(0,0)} \backslash\left(\bigcup_{k=1}^{N} D_{k} \cup \partial D_{k}\right)
$$

the connected domain obtained by removing of the inclusions from the cell $Q_{(0,0)}$.

Let us consider the problem of determination of the effective conductivity of an unbounded doubly periodic composite material with matrix

$$
D_{\text {matrix }}=\bigcup_{m_{1}, m_{2}}\left(\left(D_{0} \cup \partial Q_{(0,0)}\right)+m_{1}+\imath m_{2}\right)
$$

and inclusions

$$
D_{i n c}=\bigcup_{m_{1}, m_{2}} \bigcup_{k=1}^{N}\left(D_{k}+m_{1}+\imath m_{2}\right)
$$


occupied by materials of conductivities $\lambda_{m}>0$ and $\lambda_{k}>0$, respectively. This problem is equivalent to the determination of the potential of the corresponding fields, i.e., a function $T$ satisfying the Laplace equation in each component of the composite material,

$$
\Delta T(z)=0, \quad z \in D_{\text {matrix }} \cup D_{\text {inc }},
$$

which have to satisfy the following boundary conditions on all $\partial D_{k}, k=$ $1,2, \ldots, N$ :

$$
\begin{gathered}
T_{m}(t)=T_{k}(t), \\
\lambda_{m} \frac{\partial T_{m}}{\partial n}(t)-\lambda_{k} \frac{\partial T_{k}}{\partial n}(t)=\gamma \frac{\partial^{2} T_{k}}{\partial s^{2}}(t), \quad t \in \bigcup_{m_{1}, m_{2}} \partial D_{k},
\end{gathered}
$$

where $\gamma<0$ is a given parameter, the vector $n=\left(n_{1}, n_{2}\right)$ is the outward unit normal vector to $\partial D_{k}$,

$$
\frac{\partial}{\partial n}=n_{1} \frac{\partial}{\partial x}+n_{2} \frac{\partial}{\partial y}
$$

is the normal derivative, while

$$
\frac{\partial}{\partial s}=-n_{2} \frac{\partial}{\partial x}+n_{1} \frac{\partial}{\partial y}
$$

is the tangent derivative, and

$$
T_{m}(t):=\lim _{z \rightarrow t, z \in D_{0}} T(z), \quad T_{k}(t):=\lim _{z \rightarrow t, z \in D_{k}} T(z) .
$$

The conditions (2.2)-(2.3) form the so-called stiff imperfect contact conditions.

In addition, we assume that the heat flux is periodic on $y$. Thus,

$$
\lambda_{m} \frac{\partial T_{m}}{\partial y}\left(x, \frac{1}{2}\right)=\lambda_{m} \frac{\partial T_{m}}{\partial y}\left(x,-\frac{1}{2}\right)=-A \sin \theta+q_{1}(x),
$$

where $A$ is the intensity of an external flux. The heat flux is also periodic on $x$, and, consequently,

$$
\lambda_{m} \frac{\partial T_{m}}{\partial x}\left(-\frac{1}{2}, y\right)=\lambda_{m} \frac{\partial T_{m}}{\partial x}\left(\frac{1}{2}, y\right)=-A \cos \theta+q_{2}(y) .
$$


To complement the average flux conditions at infinity, the latter immediately proves that the equalities

$$
\int_{-1 / 2}^{1 / 2} q_{j}(\xi) d \xi=0
$$

are valid for the unknown functions $q_{j}(j=1,2)$. As a result of (2.6) and (2.7), the heat flux has a zero mean value along the boundary of the cell

$$
\int \frac{\partial T_{m}(s)}{\partial n} d s=0, \quad \int_{\partial Q_{\left(m_{1}, m_{2}\right)}+m_{1}+\imath m_{2}} \frac{\partial T_{m}(s)}{\partial n} d s=0 .
$$

The condition (2.9) is a consequence of the fact that no source (sink) exists in the cells.

\section{Solvability result and effective properties}

We introduce complex potentials $\varphi(z)$ and $\varphi_{k}(z)$ which are analytic in $D_{0}$ and $D_{k}$, and continuously differentiable in the closures of $D_{0}$ and $D_{k}$, respectively, by using the following relations

$$
T(z)=\left\{\begin{array}{l}
\operatorname{Re}(\varphi(z)+B z), z \in D_{\text {matrix }} \\
\frac{2 \lambda_{m}}{\lambda_{m}+\lambda_{k}} \operatorname{Re} \varphi_{k}(z), z \in D_{\text {inc }}
\end{array}\right.
$$

where $B$ is an unknown constant belonging to $\mathbb{C}$. Besides, we assume that the real part of $\varphi$ is doubly periodic in $D_{0}$, i.e.,

$$
\operatorname{Re} \varphi(z+1)-\operatorname{Re} \varphi(z)=0, \quad \operatorname{Re} \varphi(z+\imath)-\operatorname{Re} \varphi(z)=0 .
$$

Note that in general the imaginary part of $\varphi$ is not doubly periodic in $D_{0}$.

It is shown in [4] that $\varphi$ is a single-valued function in $D_{\text {matrix }}$. The harmonic conjugate to $T$ is a function $v$ which has the following form:

$$
v(z)=\left\{\begin{array}{l}
\operatorname{Im}(\varphi(z)+B z), z \in D_{\text {matrix }}, \\
\frac{2 \lambda_{m}}{\lambda_{m}+\lambda_{k}} \operatorname{Im} \varphi_{k}(z), z \in D_{\text {inc }},
\end{array}\right.
$$

with the same unknown constant $B$. 
For the determination of the flux $\nabla u(x, y)$, we introduce the derivatives of the complex potentials:

$$
\begin{array}{ll}
\psi(z):=\frac{\partial \varphi}{\partial z}=\frac{\partial T_{m}}{\partial x}-\imath \frac{\partial T_{m}}{\partial y}-B, & z \in D_{0}, \\
\psi_{k}(z):=\frac{\partial \varphi_{k}}{\partial z}=\frac{\lambda_{m}+\lambda_{k}}{2 \lambda_{m}}\left(\frac{\partial T_{k}}{\partial x}-\imath \frac{\partial T_{k}}{\partial y}\right), & z \in D_{k} .
\end{array}
$$

According to (2.4) and (3.3), the boundary value of the normal derivative can be written in the form

$$
\begin{aligned}
\frac{\partial T_{k}(t)}{\partial n} & =\operatorname{Re}\left(\left(n_{1}+\imath n_{2}\right)\left(\frac{\partial T_{k}}{\partial x}-\imath \frac{\partial T_{k}}{\partial y}\right)\right) \\
& =\frac{2 \lambda_{m}}{r_{k}\left(\lambda_{m}+\lambda_{i}\right)} \operatorname{Re}\left[\left(t-a_{k}\right)\left(\varphi_{k}\right)^{\prime}(t)\right]
\end{aligned}
$$

where the normal vector $n$ to the disk $\left|t-a_{k}\right|=r_{k}$ is written as $n=\frac{t-a_{k}}{r_{k}}$. Using (2.5) and (3.3), the boundary value of the tangent derivative can be found as

$$
\begin{aligned}
\frac{\partial T_{k}(t)}{\partial s} & =\operatorname{Im}\left(-\left(n_{1}+\imath n_{2}\right)\left(\frac{\partial T_{k}}{\partial x}-\imath \frac{\partial T_{k}}{\partial y}\right)\right) \\
& =-\frac{2 \lambda_{m}}{r_{k}\left(\lambda_{m}+\lambda_{i}\right)} \operatorname{Im}\left[\left(t-a_{k}\right)\left(\varphi_{k}\right)^{\prime}(t)\right] .
\end{aligned}
$$

Applying the Cauchy-Riemann equations

$$
\frac{\partial T_{m}}{\partial n}=\frac{\partial v_{m}}{\partial s}, \quad \frac{\partial T_{k}}{\partial n}=\frac{\partial v_{k}}{\partial s},
$$

the equality (2.3) can be written as

$$
\lambda_{m} \frac{\partial v_{m}}{\partial s}(t)-\lambda_{k} \frac{\partial v_{k}}{\partial s}(t)=\gamma \frac{\partial^{2} T_{k}}{\partial s^{2}}, \quad\left|t-a_{k}\right|=r_{k} .
$$

Integrating the last equality on $s$, we arrive at the relation

$$
\lambda_{m} v_{m}(t)-\lambda_{k} v_{k}(t)=\gamma \frac{\partial T_{k}}{\partial s}+c
$$

where $c$ is an arbitrary constant. We put $c=0$ since the imaginary part of the function $\varphi$ is determined up to an additive constant which does not 
impact on the form of $T$. Using (3.2) and (3.5), we have

$$
\operatorname{Im} \varphi(t)=-\operatorname{Im} B t+\frac{2 \lambda_{k}}{\lambda_{m}+\lambda_{k}} \operatorname{Im} \varphi_{k}(t)-\frac{2 \gamma}{r_{k}\left(\lambda_{m}+\lambda_{k}\right)} \operatorname{Im}\left[\left(t-a_{k}\right)\left(\varphi_{k}\right)^{\prime}(t)\right] .
$$

Using (3.1), we are able to write the equality (2.2) in the following form:

$$
\operatorname{Re} \varphi(t)=-\operatorname{Re} B t+\frac{2 \lambda_{m}}{\lambda_{m}+\lambda_{m}} \operatorname{Re} \varphi_{k}
$$

Adding the relation (3.10) and (3.9) multiplied by $\imath$, and using $\operatorname{Re} \varphi_{k}=\frac{\varphi_{k}+\overline{\varphi_{k}}}{2}$, $\operatorname{Im} \varphi_{k}=\frac{\varphi_{k}-\overline{\varphi_{k}}}{2 \imath}, t-a_{k}=\frac{r_{k}^{2}}{\overline{t-a_{k}}}$, we rewrite the conditions (2.2) and (2.3) in terms of the complex potentials $\varphi(z)$ and $\varphi_{k}(z)$ :

$\varphi(t)=\varphi_{k}(t)-\rho_{k} \overline{\varphi_{k}(t)}+\mu_{k}\left(t-a_{k}\right)\left(\varphi_{k}\right)^{\prime}(t)-\mu_{k} \frac{r_{k}^{2}}{t-a_{k}} \overline{\left(\varphi_{k}\right)^{\prime}(t)}-B t,\left|t-a_{k}\right|=r_{k}$,

where $\rho_{k}=\frac{\lambda_{k}-\lambda_{m}}{\lambda_{m}+\lambda_{k}}$ and

$$
\mu_{k}=-\frac{\gamma}{r_{k}\left(\lambda_{m}+\lambda_{k}\right)}
$$

Representing the function $\varphi$ in the form $\varphi(z)=\sum_{l=0}^{\infty} \alpha_{k}\left(z-a_{k}\right)^{l},\left|z-a_{k}\right| \leq r_{k}$, and by using the relation $t=\frac{r_{k}^{2}}{t-a_{k}}+a_{k}$ on the boundary $\left|t-a_{k}\right|=r_{k}$, one can get

$$
[\overline{\varphi(t)}]^{\prime}=-\left(\frac{r_{k}}{t-a_{k}}\right)^{2} \overline{\varphi^{\prime}(t)}, \quad\left[\overline{\varphi^{\prime}(t)}\right]^{\prime}=-\left(\frac{r_{k}}{t-a_{k}}\right)^{2} \overline{\psi^{\prime}(t)},\left|t-a_{k}\right|=r_{k} .
$$

Thus, after differentiating (3.11), we arrive at the following $R$-linear boundary value problem on each contour $\left|t-a_{k}\right|=r_{k}, k=1,2, \ldots, N$,

$$
\begin{aligned}
\psi(t)= & \left(1+\mu_{k}\right) \psi_{k}(t)+\left(\rho_{k}+\mu_{k}\right)\left(\frac{r_{k}}{t-a_{k}}\right)^{2} \overline{\psi_{k}(t)}+\mu_{k}\left(t-a_{k}\right) \psi_{k}^{\prime}(t) \\
& +\mu_{k} \frac{r_{k}^{4}}{\left(t-a_{k}\right)^{3}} \overline{\psi_{k}^{\prime}(t)}-B
\end{aligned}
$$

with the unknown constant $B$.

In order to find the functions $\psi$ and $\psi_{k}$ inside the matrix and inclusions, respectively, we need results from [5]. 
Let the functions $\widetilde{\varphi}^{(1)}$ and $\widetilde{\varphi}_{k}^{(1)}$ be solutions of an auxiliary problem (2.1)(2.3) with a constant jump corresponding to the external field applied in the $x$-direction

$$
T(z+1)=T(z)+1, \quad T(z+\imath)=T(z)
$$

(instead of conditions (2.6), (2.7)) and functions $\widetilde{\varphi}^{\perp}$ and $\widetilde{\varphi}_{k}^{\perp}$ be solutions of an auxiliary problem (2.1)-(2.3) with a constant jump corresponding to the external field applied in the $y$-direction

$$
T(z+1)=T(z), \quad T(z+\imath)=T(z)-1
$$

(instead of conditions (2.6),$(2.7)$ ). Then the following results hold:

Theorem 3.1 Let $T_{m}=T_{m}(x, y)$ and $T_{k}=T_{k}(x, y)$ be the solution of the problem (2.2)-(2.3), (2.6) and (2.7). The temperature distribution can be found up to an arbitrary constant and is defined in the form (3.1), where

$$
\begin{aligned}
B & =\frac{-A \cos \theta}{\lambda_{m}(I+1)}-\frac{A \sin \theta}{\lambda_{m}\left(I^{\perp}-1\right)} \imath, \\
\varphi(z) & =\frac{-A \cos \theta}{\lambda_{m}(I+1)} \widetilde{\varphi}^{(1)}(z)-\frac{A \sin \theta}{\lambda_{m}\left(I^{\perp}-1\right)} \widetilde{\varphi}^{\perp}(-\imath z), \\
\varphi_{k}(z) & =\frac{-A \cos \theta}{\lambda_{m}(I+1)} \widetilde{\varphi}_{k}^{(1)}(z)-\frac{A \sin \theta}{\lambda_{m}\left(I^{\perp}-1\right)} \widetilde{\varphi}_{k}^{\perp}(-\imath z) .
\end{aligned}
$$

Theorem 3.2 Let $T_{m}=T_{m}(x, y)$ and $T_{k}=T_{k}(x, y)$ be the solution of the problem (2.2)-(2.3), (2.6) and (2.7). The temperature flux is defined in the following form:

$$
\frac{\partial T(x, y)}{\partial x}-\imath \frac{\partial T(x, y)}{\partial y}=\left\{\begin{array}{l}
\psi(z)+B, z=x+\imath y \in D_{\text {matrix }} \\
\frac{2 \lambda_{m}}{\lambda_{m}+\lambda_{k}} \psi_{k}(z), z=x+\imath y \in D_{i n c}
\end{array}\right.
$$

with

$$
B=\frac{-A \cos \theta}{\lambda_{m}(I+1)}-\frac{A \sin \theta}{\lambda_{m}\left(I^{\perp}-1\right)} \imath,
$$

and

$$
\begin{aligned}
\psi(z) & :=\frac{-A \cos \theta}{\lambda_{m}(I+1)} \widetilde{\psi}^{(1)}(z)+\imath \frac{A \sin \theta}{\lambda_{m}\left(I^{\perp}-1\right)} \widetilde{\psi}^{\perp}(-\imath z), \quad z \in D_{\text {matrix }}, \\
\psi_{k}(z) & :=\frac{-A \cos \theta}{\lambda_{m}(I+1)} \widetilde{\psi}_{k}^{(1)}(z)+\imath \frac{A \sin \theta}{\lambda_{m}\left(I^{\perp}-1\right)} \widetilde{\psi}_{k}^{\perp}(-\imath z), \quad z \in D_{i n c},
\end{aligned}
$$


where the functions $\widetilde{\psi}^{(1)}, \widetilde{\psi}^{\perp}, \widetilde{\psi}_{k}^{(1)}$ and $\widetilde{\psi}_{k}^{\perp}$ are derivatives of the functions $\widetilde{\varphi}^{(1)}, \widetilde{\varphi}^{\perp}, \widetilde{\varphi}_{k}^{(1)}$ and $\widetilde{\varphi}_{k}^{\perp}$, respectively.

The proofs of these theorems are direct consequences of the results obtained in [5], where an analogous to (3.11), (3.14) problems are analytically solved using the method of functional equations.

Finding the functions $\widetilde{\psi}^{(1)}$ and $\widetilde{\psi}_{k}^{(1)}, k=1, \ldots, N$, is reduced to the solution of a system of functional equations (cf. [5] )

$$
\begin{aligned}
\widetilde{\psi}_{k}(z)= & -\frac{\mu_{k}}{1+\mu_{k}}\left(z-a_{k}\right) \widetilde{\psi}_{k}^{\prime}(z) \\
& +\frac{1}{1+\mu_{k}} \sum_{m \neq k}^{N} \sum_{l=0}^{\infty}\left(\rho_{m}+\mu_{m}\right) \overline{\widetilde{\psi}_{l m}} r_{m}^{2(l+1)} E_{l+2}\left(z-a_{m}\right) \\
& +\frac{\rho_{k}+\mu_{k}}{1+\mu_{k}} \sum_{l=0}^{\infty} \overline{\widetilde{\psi}_{l k}} r_{k}^{2(l+1)} \sigma_{l+2}\left(z-a_{k}\right) \\
& +\frac{1}{1+\mu_{k}} \sum_{m \neq k}^{N} \sum_{l=1}^{\infty} \mu_{m} \widetilde{\psi_{l m}} l r_{m}^{2(l+1)} E_{l+2}\left(z-a_{m}\right) \\
& +\frac{\mu_{k}}{1+\mu_{k}} \sum_{l=1}^{\infty} \overline{\widetilde{\psi}_{l k}} l r_{k}^{2(l+1)} \sigma_{l+2}\left(z-a_{k}\right)+\frac{1}{1+\mu_{k}}, \\
\widetilde{\psi}(z)= & \sum_{m=1}^{N} \sum_{l=0}^{\infty}\left(\rho_{m}+\mu_{m}\right) \widetilde{\widetilde{\psi}_{l m}} r_{m}^{2(l+1)} E_{l+2}\left(z-a_{m}\right) \\
& +\sum_{m=1}^{N} \sum_{l=1}^{\infty} \overline{\widetilde{\psi}_{l m}} l r_{m}^{2(l+1)} E_{l+2}\left(z-a_{m}\right) .
\end{aligned}
$$

Theorem 3.3 For the parameters $\mu_{k}>0, k=1, \ldots, N$, the equation (3.16) has a unique solution in $\mathcal{H}^{2}$.

Here, $\mathcal{H}^{2}\left(D_{k}\right)$ is the generalized Hardy space spaces of analytic functions on $D_{k}$ satisfying the condition

$$
\sup _{0<r<r_{k}} \int_{0}^{2 \pi}\left|\psi_{k}\left(r e^{\imath \theta}+a_{k}\right)\right|^{2} d \theta<\infty
$$


and correspondingly endowed with the norm

$$
\left\|\psi_{k}\right\|_{\mathcal{H}^{2}\left(D_{k}\right)}^{2}:=\sup _{0<r<r_{k}} \int_{0}^{2 \pi}\left|\psi_{k}\left(r e^{\imath \theta}+a_{k}\right)\right|^{2} d \theta .
$$

The proof of this theorem completely repeats the proof of an analogous theorem in [8] for the case of soft imperfect conditions on the boundaries of components.

Remark 3.4 It directly follows from Theorem 3.3 and (3.12) that the system (3.16), (3.17) always has a unique solution as since $\gamma<0$ according to the statement of the problem.

All arguments for the functions $\widetilde{\psi}^{(1)}$ and $\widetilde{\psi}_{k}^{(1)}$ are valid for the functions $\widetilde{\psi}^{\perp}$ and $\widetilde{\psi_{k}^{\perp}}$.

Now the components of the effective conductivity tensor

$$
\Lambda_{e}=\left(\begin{array}{cc}
\lambda_{e}^{x} & \lambda_{e}^{x y} \\
\lambda_{e}^{x y} & \lambda_{e}^{y}
\end{array}\right) .
$$

can be found from the well-known equation

$$
\langle\mathbf{q}\rangle=-\Lambda_{e} \cdot\langle\nabla T\rangle
$$

where $\langle\mathbf{q}\rangle=\left(\mathfrak{q}_{1}, \mathfrak{q}_{2}\right)$ is the average flux, and $\langle\nabla T\rangle=\left(T_{1}, T_{2}\right)$ is the average temperature gradient with

$$
\begin{aligned}
& \mathfrak{q}_{1}=\lambda_{m} \iint_{D_{0}} \frac{\partial T_{m}}{\partial x} d x d y+\sum_{k=1}^{N} \lambda_{k} \iint_{D_{k}} \frac{\partial T_{k}}{\partial x} d x d y \\
& \mathfrak{q}_{2}=\lambda_{m} \iint_{D_{0}} \frac{\partial T_{m}}{\partial y} d x d y+\sum_{k=1}^{N} \lambda_{k} \iint_{D_{k}} \frac{\partial T_{k}}{\partial y} d x d y
\end{aligned}
$$

and

$$
T_{1}=\iint_{D_{0}} \frac{\partial T_{m}}{\partial x} d x d y+\sum_{k=1}^{N} \iint_{D_{k}} \frac{\partial T_{k}}{\partial x} d x d y
$$




$$
T_{2}=\iint_{D_{0}} \frac{\partial T_{m}}{\partial y} d x d y+\sum_{k=1}^{N} \iint_{D_{k}} \frac{\partial T_{k}}{\partial y} d x d y .
$$

In order to optimize the forthcoming numerical calculations, we transform the integrals above in a more convenient form, by using the first Green's formula,

$$
\iint_{U}(\psi \Delta \varphi+\nabla \varphi \cdot \nabla \psi) d V=\oint_{\partial U} \psi(\nabla \varphi \cdot \mathbf{n}) d S
$$

with $\psi=x$ or $\psi=y$ and $\varphi(x, y)=T_{m}$ in $D_{0}\left(\right.$ or $\varphi(x, y)=T_{k}$ in the respective domain $D_{k}$ ):

$$
\iint_{D_{0}} \frac{\partial T}{\partial x} d x d y=\oint_{\partial D_{0}} x \frac{\partial T}{\partial n} d s=\oint_{\partial Q_{(0,0)}} x \frac{\partial T}{\partial n} d s-\sum_{k=1}^{N} \oint_{\partial D_{k}} x \frac{\partial T_{k}}{\partial n} d s
$$

where the curves $\partial Q_{(0,0)}$ and $\partial D_{k}$ are oriented in the counterclockwise direction. The first integral can be directly computed with use of (2.6), (2.7) and (2.8)

$$
\begin{gathered}
\oint_{\partial Q_{(0,0)}} x \frac{\partial T}{\partial n} d s=\frac{1}{\lambda_{m}}\left(\int_{-1 / 2}^{1 / 2} x(-A \sin \theta) d x-\int_{-1 / 2}^{1 / 2} x(-A \sin \theta) d x+\right. \\
\left.\frac{1}{2} \int_{-1 / 2}^{1 / 2}(-A \cos \theta) d y+\frac{1}{2} \int_{-1 / 2}^{1 / 2}(-A \cos \theta) d y\right)=-\frac{A}{\lambda_{m}} \cos \theta .
\end{gathered}
$$

Repeating the same line of the reasoning with $\psi=y$ and $\varphi(x, y)=T_{m}$ in $D_{0}\left(\right.$ or $\varphi(x, y)=T_{k}$ in the respective domain $D_{k}$ ) with first Green's formula (3.23), we have

$$
\oint_{\partial Q_{(0,0)}} y \frac{\partial T}{\partial n} d s=-\frac{A}{\lambda_{m}} \sin \theta .
$$

Thus using the Green formula (3.23) and (2.3), we have

$$
\begin{aligned}
\mathfrak{q}_{1} & =\lambda_{m}\left(\oint_{\partial Q_{(0,0)}} x \frac{\partial T}{\partial n} d s-\sum_{k=1}^{N} \oint_{\partial D_{k}} x \frac{\partial T_{k}}{\partial n} d s\right)+\sum_{k=1}^{N} \lambda_{k} \oint_{\partial D_{k}} x \frac{\partial T_{k}}{\partial n} d s \\
& =-A \cos \theta-\gamma \sum_{k=1}^{N} \oint_{\partial D_{k}} x \frac{\partial^{2} T_{k}}{\partial s^{2}} d s .
\end{aligned}
$$




$$
\mathfrak{q}_{2}=-A \sin \theta-\gamma \sum_{k=1}^{N} \oint_{\partial D_{k}} y \frac{\partial^{2} T_{k}}{\partial s^{2}} d s .
$$

According to the same arguments as above, we have

$$
\begin{aligned}
& T_{1}=-\frac{A \cos \theta}{\lambda_{m}}+\sum_{k=1}^{N}\left(1-\frac{\lambda_{k}}{\lambda_{m}}\right) \iint_{D_{k}} \frac{\partial T_{k}}{\partial x} d x d y-\frac{\gamma}{\lambda_{m}} \sum_{k=1}^{N} \oint_{\partial D_{k}} x \frac{\partial^{2} T_{k}}{\partial s^{2}} d s, \\
& T_{2}=-\frac{A \sin \theta}{\lambda_{m}}+\sum_{k=1}^{N}\left(1-\frac{\lambda_{k}}{\lambda_{m}}\right) \iint_{D_{k}} \frac{\partial T_{k}}{\partial y} d x d y-\frac{\gamma}{\lambda_{m}} \sum_{k=1}^{N} \oint_{\partial D_{k}} y \frac{\partial^{2} T_{k}}{\partial s^{2}} d s .
\end{aligned}
$$

Combining these values together with use of (3.3) and the mean value theorem for harmonic functions, we have

$$
\begin{aligned}
T_{1}-\imath T_{2}= & \frac{-A e^{-\imath \theta}}{\lambda_{m}}+2 \sum_{k=1}^{N} \frac{\lambda_{m}-\lambda_{k}}{\lambda_{m}+\lambda_{k}} \iint_{D_{k}} \psi_{k}(z) d x d y \\
& -\frac{\gamma}{\lambda_{m}} \sum_{k=1}^{N} \oint_{\partial D_{k}}(x-\imath y) \frac{\partial^{2} T_{k}}{\partial s^{2}} d s \\
= & \frac{-A e^{-\imath \theta}}{\lambda_{m}}-2 \pi \sum_{k=1}^{N} \rho_{k} r_{k}^{2} \psi_{k}\left(a_{k}\right)-\frac{\gamma}{\lambda_{m}} \sum_{k=1}^{N} \oint_{\partial D_{k}}(x-\imath y) \frac{\partial^{2} T_{k}}{\partial s^{2}} d s .
\end{aligned}
$$

\section{Numerical results and discussions}

In this section, we present numerical calculations of the material characteristics such as temperature, flux and the effective conductivity using the Maple 14 software.

First, we discuss an accuracy of calculations and choose a non-symmetrical configuration of non-overlapping inclusions with the centers

$$
a_{1}=-0.18+0.2 \imath, a_{2}=0.33-0.34 \imath, a_{3}=0.33+0.35 \imath, a_{4}=-0.18-0.2 \imath
$$

and the same radius $r_{k}=R$ of value 0.145 . In this case some of the inclusions are situated very close to inclusions of the adjoin cells. For symmetrical configurations of the inclusions or smaller radius, the accuracy is higher. Therefore, we choose that configuration to check the accuracy of the calculations 
Table 1: Temperature in two points of the model for different numbers of $M$, while other problem parameters are: $\theta=0, \gamma=-0.1, R=0.145, \lambda_{m}=1$, $\lambda_{k}=0.01$ and the configuration of the inclusions being defined by (4.1).

\begin{tabular}{|c|c|c|}
\hline$M$ & $T(0)$ & $T\left(a_{1}\right)$ \\
\hline 0 & 0.01027845 & -0.19751811 \\
1 & 0.01005208 & -0.19752105 \\
2 & 0.01003072 & -0.19749178 \\
3 & 0.01003070 & -0.19748953 \\
4 & 0.01003221 & -0.19749164 \\
5 & 0.01003308 & -0.19749199 \\
6 & 0.01003318 & -0.19749186 \\
7 & 0.01003320 & -0.19749178 \\
\hline
\end{tabular}

Table 2: The flux components for different numbers of $M$, while other problem parameters are: $\theta=0, \gamma=-0.1, R=0.145, \lambda_{m}=1, \lambda_{k}=0.01$ and the configuration of the inclusions being defined by (4.1).

\begin{tabular}{|c|c|c|c|c|}
\hline$M$ & $Q_{x}^{(m)}(0)$ & $Q_{y}^{(m)}(0)$ & $Q_{x}^{(1)}\left(a_{1}\right)$ & $Q_{y}^{(1)}\left(a_{1}\right)$ \\
\hline 0 & 1.06384804 & 0.00074222 & 0.01247853 & 0.00001216 \\
1 & 1.06432063 & 0.00074695 & 0.01247441 & 0.00001194 \\
2 & 1.06512488 & 0.00075325 & 0.01247367 & 0.00001206 \\
3 & 1.06515122 & 0.00075132 & 0.01247388 & 0.00001206 \\
4 & 1.06517143 & 0.00075267 & 0.01247383 & 0.00001205 \\
5 & 1.06516444 & 0.00075249 & 0.01247381 & 0.00001205 \\
6 & 1.06516139 & 0.00075247 & 0.01247381 & 0.00001205 \\
7 & 1.06516090 & 0.00075247 & 0.01247381 & 0.00001205 \\
\hline
\end{tabular}

in the worst situation. We suppose that a heat flux of fixed intensity $A=-1$ flows in different directions with respect to the main axis depending on the angle $\theta$. The conductivity of the matrix is $\lambda_{m}=1$, and the conductivity of the inclusions $\lambda_{k}$ take different values. Values of the parameter $\gamma$ will be chosen in accordance with Remark 3.4 .

Second, we calculate the temperature and flux components in the matrix 
point $z=0$ when the flux components in any point of the matrix can be found as (cf. (3.15))

$$
Q_{x}^{(m)}(z)=\lambda_{m} \cdot \operatorname{Re}(\psi(z)+B), \quad Q_{y}^{(m)}(z)=-\lambda_{m} \cdot \operatorname{Im}(\psi(z)+B),
$$

and in the center $a_{k}$ of the $k$-inclusion

$$
\begin{gathered}
Q_{x}^{(k)}\left(a_{k}\right) \equiv \lambda_{k} \frac{\partial T_{k}\left(a_{k}\right)}{\partial x}=\frac{2 \lambda_{k} \lambda_{m}}{\lambda_{m}+\lambda_{k}} \cdot \operatorname{Re} \psi_{k}\left(a_{k}\right), \\
Q_{y}^{(k)}\left(a_{k}\right) \equiv \lambda_{k} \frac{\partial T_{k}\left(a_{k}\right)}{\partial y}=-\frac{2 \lambda_{k} \lambda_{m}}{\lambda_{m}+\lambda_{k}} \cdot \operatorname{Im} \psi_{k}\left(a_{k}\right) .
\end{gathered}
$$

A solution $\psi$ and $\psi_{k}$ is found in terms of the Taylor series (cf. [5]).

Computations are given for the first eight consecutive values of the number $M(M=0,1, \ldots, 7)$ showing how many terms are selected for computations in the Taylor series. The results are presented in the Table 1.2 .

Computations show that taking $M=7$ the accuracy is between five or six valid units depending on where the flux is computed.

Note that for the same configuration and parameters we get the same accuracy in case of soft imperfect contact conditions on the boundaries of material components (see [5]).

We represent the temperature distribution $T(x, y)$ in Figs. 14 for fixed parameters $\lambda_{m}=1, R=0.145$ and different $\theta=0 ; \frac{\pi}{4}, \lambda_{k}=100 ; 0.01$, $\gamma=-0.1 ;-100$.

Table 3: The components of the effective conductivity tensor $\Lambda_{e}$ for the configuration of the inclusions given in (4.1) for the material constants $\lambda_{m}=$ $1, \lambda_{k}=100, \gamma_{k}=-0.1, M=7$.

\begin{tabular}{|c|c|c|c|c|}
\hline$R$ & $\lambda_{e}^{x}$ & $\lambda_{e}^{x y}$ & $\lambda_{e}^{y x}$ & $\lambda_{e}^{y}$ \\
\hline 0.01 & 1.002243 & $3.3 \cdot 10^{-10}$ & $3.3 \cdot 10^{-10}$ & 1.002245 \\
0.05 & 1.061632 & $2.4 \cdot 10^{-7}$ & $2.4 \cdot 10^{-7}$ & 1.063012 \\
0.11 & 1.328690 & $8.0 \cdot 10^{-6}$ & $8.0 \cdot 10^{-6}$ & 1.371994 \\
0.135 & 1.530238 & $2.5 \cdot 10^{-5}$ & $2.7 \cdot 10^{-5}$ & 1.658053 \\
0.145 & 1.633773 & $4.2 \cdot 10^{-5}$ & $4.7 \cdot 10^{-5}$ & 1.837122 \\
\hline
\end{tabular}

We also show the flux distribution inside the cell $\mathrm{Q}(0,0)$ for different $\gamma$, angles and conductivities of inclusions in Figs. 58 , 
Table 4: The components of the effective conductivity tensor $\Lambda_{e}$ for the configuration of the inclusions given in (4.1) for the material constants $\lambda_{m}=$ $1, \lambda_{k}=100, \gamma_{k}=-100, M=7$.

\begin{tabular}{|c|c|c|c|c|}
\hline$R$ & $\lambda_{e}^{x}$ & $\lambda_{e}^{x y}$ & $\lambda_{e}^{y x}$ & $\lambda_{e}^{y}$ \\
\hline 0.01 & 1.000025 & $3.7 \cdot 10^{-12}$ & $3.7 \cdot 10^{-12}$ & 1.000025 \\
0.05 & 1.003023 & $1.2 \cdot 10^{-8}$ & $1.2 \cdot 10^{-8}$ & 1.003092 \\
0.11 & 1.033231 & $6.7 \cdot 10^{-7}$ & $8.3 \cdot 10^{-7}$ & 1.037712 \\
0.135 & 1.064321 & $1.6 \cdot 10^{-6}$ & $3.4 \cdot 10^{-6}$ & 1.080272 \\
0.145 & 1.081874 & $1.9 \cdot 10^{-6}$ & $6.3 \cdot 10^{-6}$ & 1.109053 \\
\hline
\end{tabular}

Table 5: The components of the effective conductivity tensor $\Lambda_{e}$ for the configuration of the inclusions given in (4.1) for the material constants $\lambda_{m}=$ $1, \lambda_{k}=0.01, \gamma_{k}=-0.1, M=7$.

\begin{tabular}{|c|c|c|c|c|}
\hline$R$ & $\lambda_{e}^{x}$ & $\lambda_{e}^{x y}$ & $\lambda_{e}^{y x}$ & $\lambda_{e}^{y}$ \\
\hline 0.01 & 0.999774 & $-2.8 \cdot 10^{-11}$ & $-2.8 \cdot 10^{-11}$ & 0.999774 \\
0.05 & 0.979191 & $4.4 \cdot 10^{-8}$ & $-2.7 \cdot 10^{-8}$ & 0.979036 \\
0.11 & 0.843782 & $-1.1 \cdot 10^{-5}$ & $1.2 \cdot 10^{-7}$ & 0.844471 \\
0.135 & 0.746494 & $-2.4 \cdot 10^{-4}$ & $9.6 \cdot 10^{-7}$ & 0.751962 \\
0.145 & 0.701535 & $-5.5 \cdot 10^{-4}$ & $1.6 \cdot 10^{-6}$ & 0.710591 \\
\hline
\end{tabular}

Table 6: The components of the effective conductivity tensor $\Lambda_{e}$ for the configuration of the inclusions given in (4.1) for the material constants $\lambda_{m}=$ $1, \lambda_{k}=0.01, \gamma_{k}=-100, M=7$.

\begin{tabular}{|c|c|c|c|c|}
\hline$R$ & $\lambda_{e}^{x}$ & $\lambda_{e}^{x y}$ & $\lambda_{e}^{y x}$ & $\lambda_{e}^{y}$ \\
\hline 0.01 & 0.9999998 & $-3.8 \cdot 10^{-14}$ & $-3.8 \cdot 10^{-14}$ & 0.9999998 \\
0.05 & 0.999968 & $-1.2 \cdot 10^{-10}$ & $-1.3 \cdot 10^{-10}$ & 0.999968 \\
0.11 & 0.999631 & $-7.2 \cdot 10^{-9}$ & $-9.6 \cdot 10^{-9}$ & 0.999581 \\
0.135 & 0.999270 & $-1.6 \cdot 10^{-8}$ & $-3.9 \cdot 10^{-8}$ & 0.999089 \\
0.145 & 0.999063 & $-1.4 \cdot 10^{-8}$ & $-7.2 \cdot 10^{-8}$ & 0.998752 \\
\hline
\end{tabular}




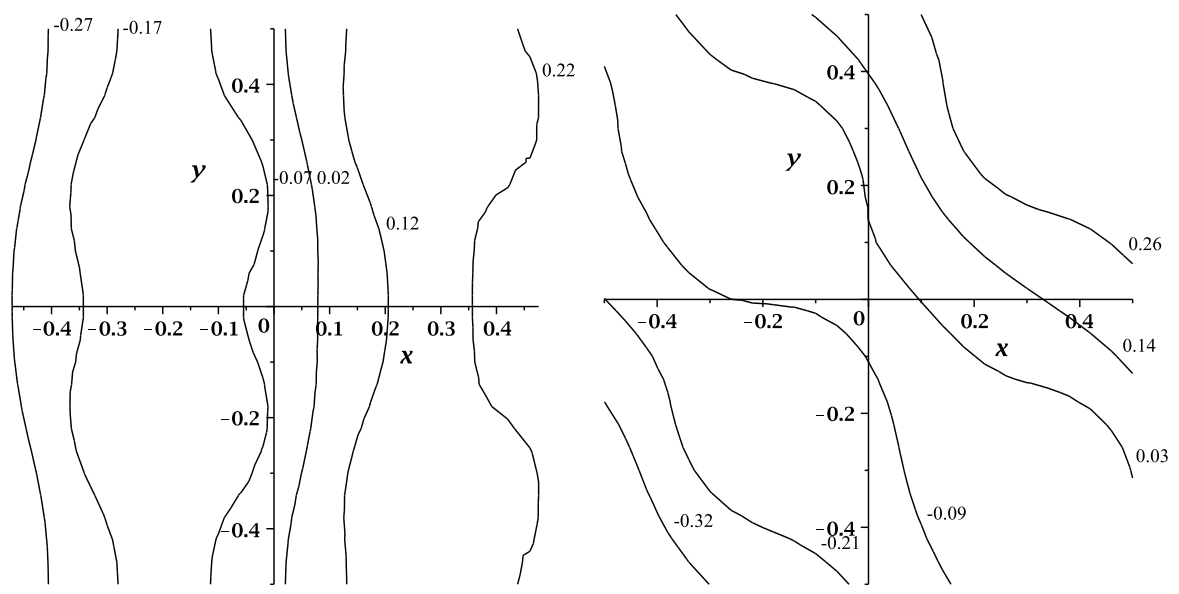

Figure 1: The temperature distribution inside $Q_{(0,0)}$ for $\lambda_{k}=100, \gamma=-0.1$, $\theta=0 ; \pi / 4$.

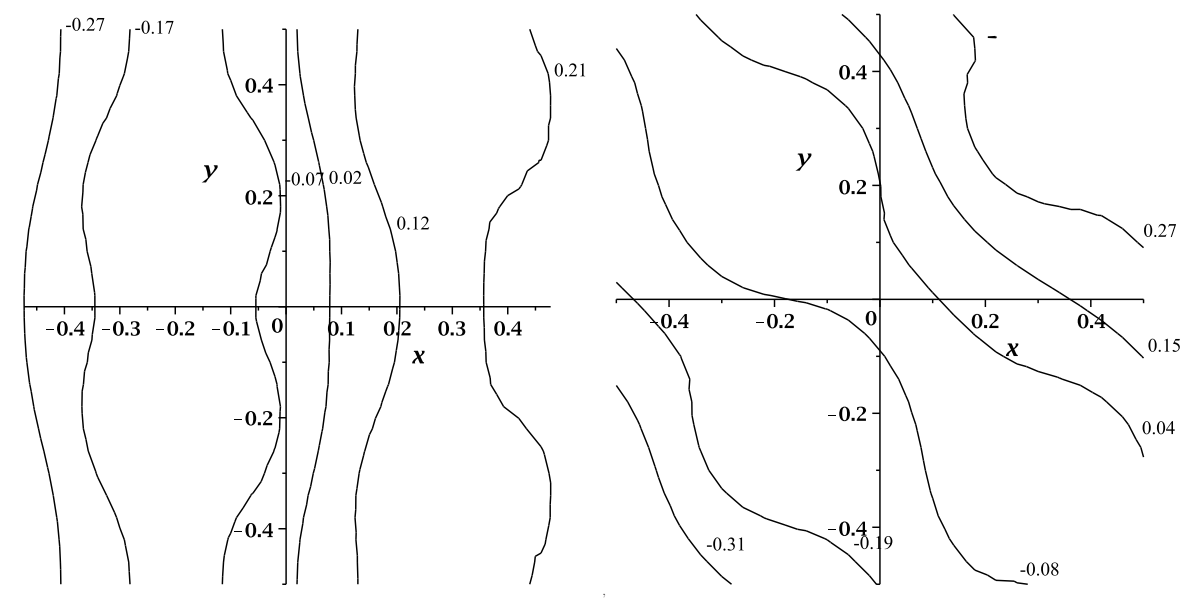

Figure 2: The temperature distribution inside $Q_{(0,0)}$ for $\lambda_{k}=100, \gamma=-100$, $\theta=0 ; \pi / 4$. 

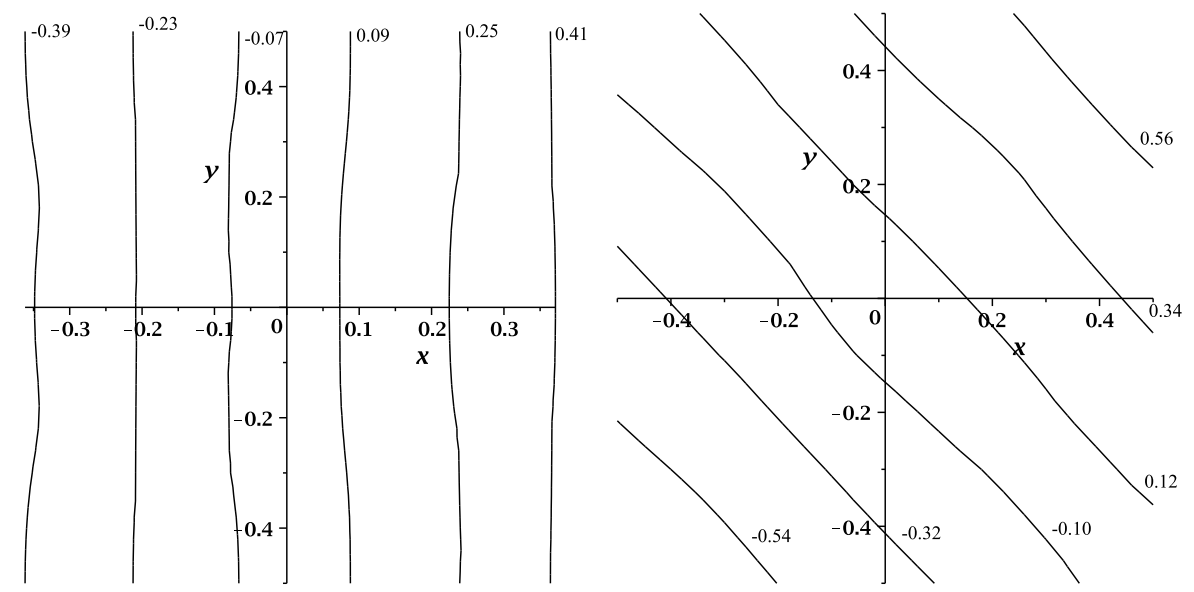

Figure 3: The temperature distribution inside $Q_{(0,0)}$ for $\lambda_{k}=0.01, \gamma=-0.1$, $\theta=0 ; \pi / 4$.
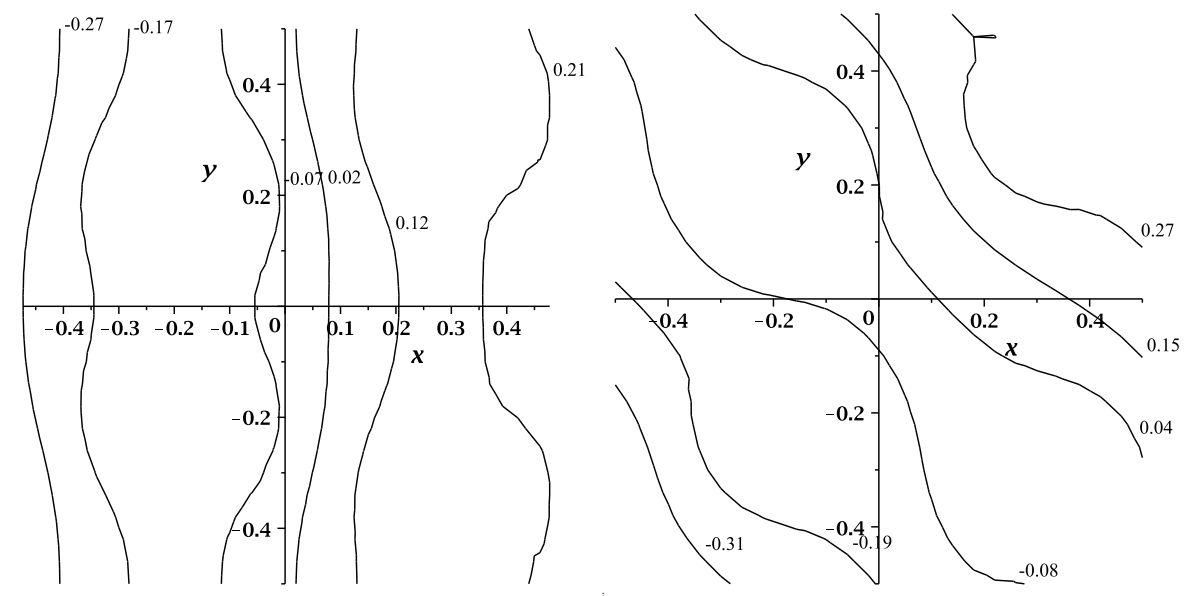

Figure 4: The temperature distribution inside $Q_{(0,0)}$ for $\lambda_{k}=0.01, \gamma=-100$, $\theta=0 ; \pi / 4$.

One can see that in most cases for fixed $\lambda_{m}=1$ the flux in the matrix is more intensive in comparison with the flux in the inclusions except the cases when values of inclusion conductivities are much more then absolute values of the parameter $\gamma$ as it occurs for $\lambda_{k}=100$ and $\gamma=-0.1$. 

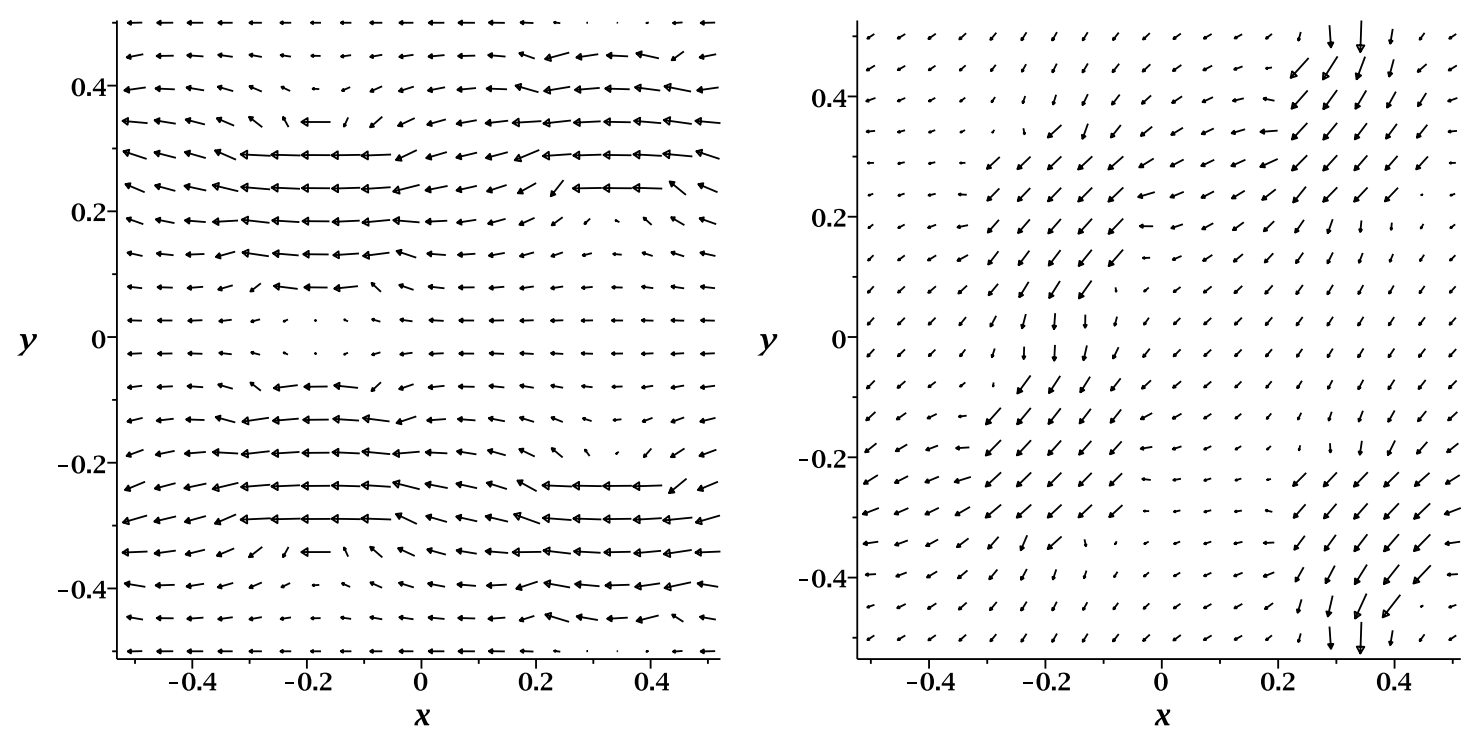

Figure 5: The flux distribution inside $Q_{(0,0)}$ for $\lambda_{k}=100, \gamma=-0.1, \theta=$ $0 ; \pi / 4$.

The values of all components of the tensor $\Lambda_{e}$ as a function on the radius $R$ are presented in Tables [3] for different parameters $\lambda_{k}$ and $\gamma$. The calculations were performed with the found accuracy between five or six valid units.

Acknowledgements. L.P. Castro is supported in part by Portuguese funds through the CIDMA - Center for Research and Development in Mathematics and Applications, and the Portuguese Foundation for Science and Technology ("FCT-Fundação para a Ciência e a Tecnologia"), within project PEstOE/MAT/UI4106/2014. D. Kapanadze and E. Pesetskaya are supported by Shota Rustaveli National Science Foundation with the grant number 31/39. The authors also would like to thank to Prof. G. Mishuris for fruitful discussions. 

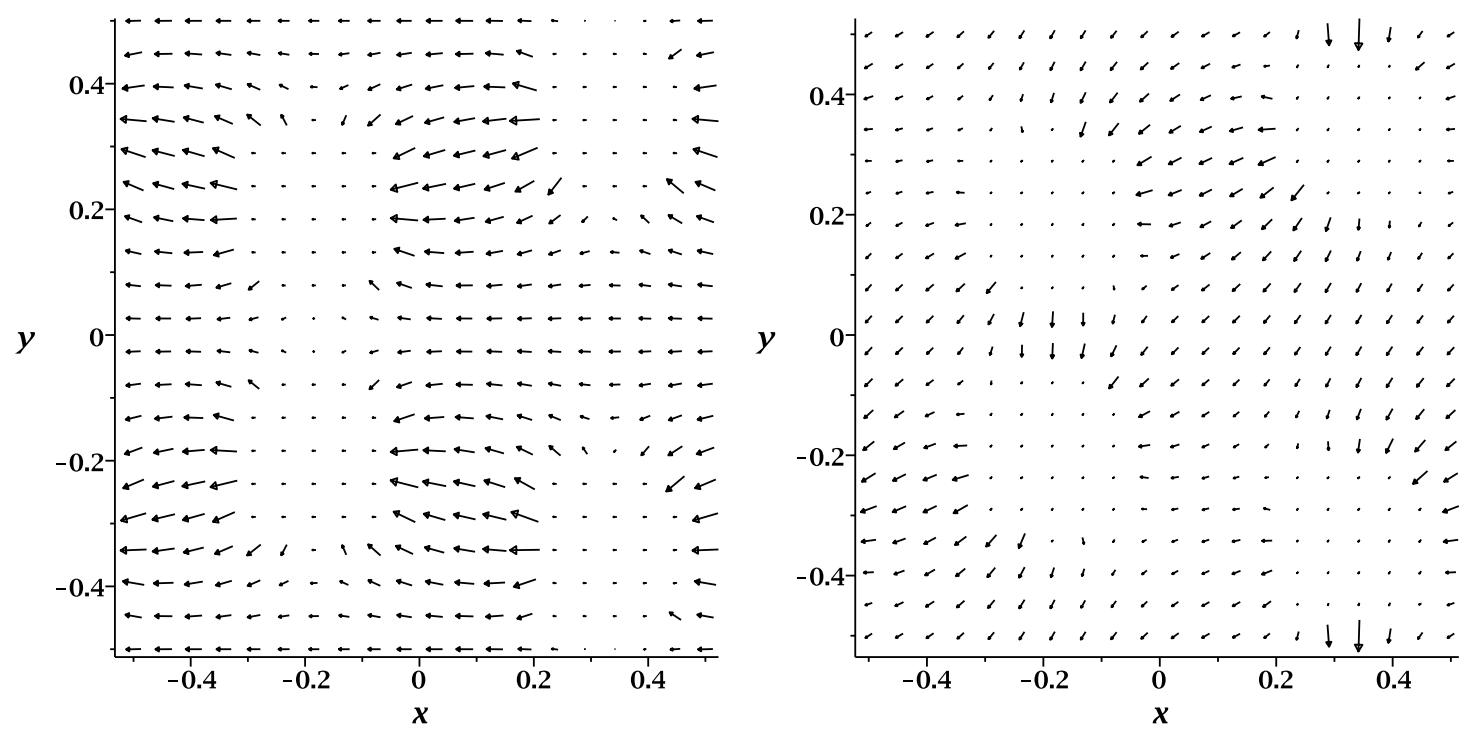

Figure 6: The flux distribution inside $Q_{(0,0)}$ for $\lambda_{k}=100, \gamma=-100, \theta=$ $0 ; \pi / 4$.

\section{References}

[1] Mityushev V. Transport properties of doubly periodic arrays of circular cylinders and optimal design problems. Applied Mathematics and Optimization. 2001; 44 (1):17-31. DOI: 10.1007/s00245-001-0013-y

[2] Berlyand L, Mityushev V. Generalized Clausius-Mossotti formula for random composite with circular fibers. Journal of Statistical Physics. 2001; 102 (1-2):115-145.

[3] Drygas P, Mityushev V. Effective conductivity of arrays of unidirectional cylinders with interfacial resistance. The Quarterly Journal of Mechanics and Applied Mathematics. 2009; 62:235-262.

[4] Kapanadze D, Mishuris G, Pesetskaya E. Improved algorithm for analytical solution of the heat conduction problem in composites. Complex Variables and Elliptic Equations. 23pp., to appear. DOI: 10.1080/17476933.2013.876418. 

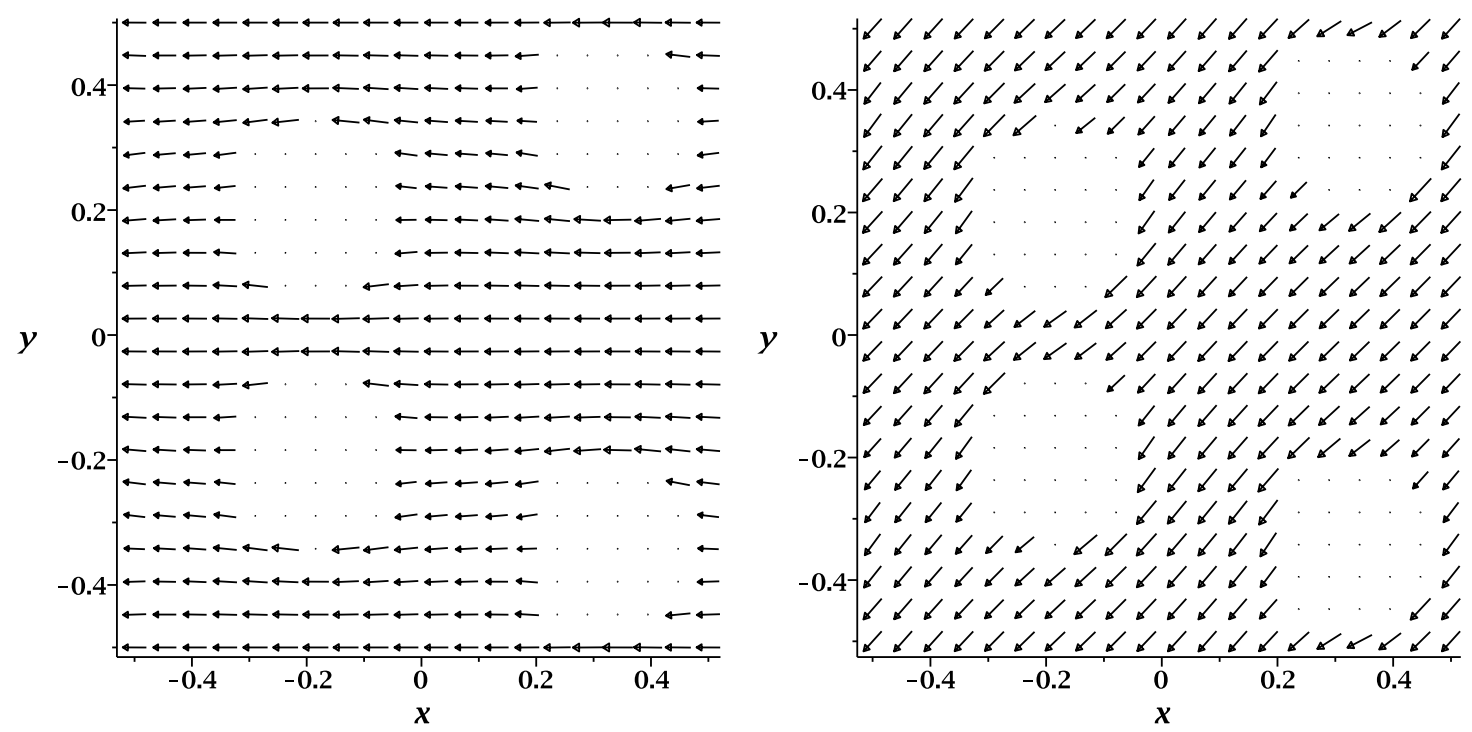

Figure 7: The flux distribution inside $Q_{(0,0)}$ for $\lambda_{k}=0.01, \gamma=-0.1, \theta=$ $0 ; \pi / 4$.

[5] Castro LP, Kapanadze D, Pesetskaya E. A heat conduction problem of 2D unbounded composites with imperfect contact conditions. Zeitschrift für Angewandte Mathematik und Mechanik. 14pp., to appear. DOI: 10.1002/zamm.201400067.

[6] Jikov VV, Kozlov SM, Olejnik OA. Homogenization of Differential Operators and Integral Functionals. Springer-Verlag: Berlin; 1994.

[7] Castro LP, Pesetskaya E. A transmission problem with imperfect contact for an unbounded multiply connected domain. Mathematical Methods in the Applied Sciences. 2010; 33:517-526. DOI: 10.1002/mma.1217.

[8] Castro LP, Pesetskaya E, Rogosin S. Effective conductivity of a composite material with non-ideal contact conditions. Complex Variables and Elliptic Equations. 2009; 54 (12):1085-1100. DOI:10.1080/17476930903275995. 

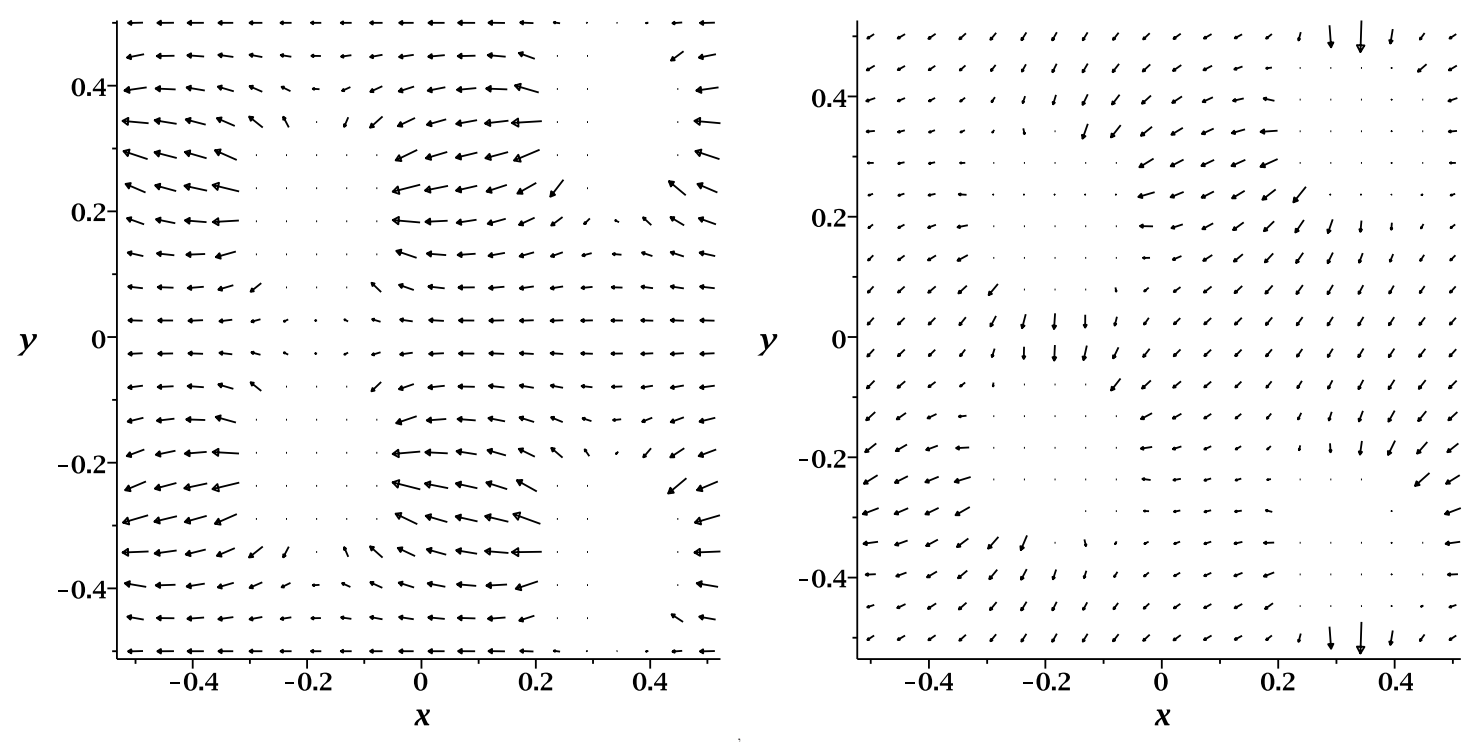

Figure 8: The flux distribution inside $Q_{(0,0)}$ for $\lambda_{k}=0.01, \gamma=-100, \theta=$ $0 ; \pi / 4$.

[9] Cheng H, Torquato S. Effective conductivity of periodic arrays of spheres with interfacial resistance. Proceedings of the Royal Society of London A. 1997; 45:145-161. DOI: 10.1098/rspa.1997.0009.

[10] Gonçalves LCC, Kołodziej JA. Determination of effective thermal conductivity in fibrous composites with imperfect contact between constituents. International Communications in Heat and Mass Transfer. 1993; 20:111-121. DOI:10.1016/0735-1933(93)90012-K.

[11] Dalla Riva M, Musolino P. A singularly perturbed nonideal transmission problem and application to the effective conductivity of a periodic composite. SIAM Journal on Applied Mathematics. 2013; 73 (1):24-46. DOI:10.1137/120886637.

[12] Béchet É, Moës N, Wohlmuth B. A stable Lagrange multiplier space for stiff interface conditions within the extended finite element method. Numerical Methods in Engineering. 2009; 78:931-954. DOI: 10.1002/nme.2515. 
[13] Teng H. Stiffness properties of particulate composites containing debonded particles. International Journal of Solids and Structures. 2010; 47 (17):2191-2200. DOI:10.1016/j.ijsolstr.2010.04.004.

[14] Benveniste Y, Miloh T. Imperfect soft and stiff interfaces in twodimensional elasticity. Mechanics of Materials. 2001; 33:309-323. DOI:10.1016/S0167-6636(01)00055-2.

[15] Mishuris GS. Mode III Interface Crack Lying at Thin Nonhomogeneous Anisotropic Interface. Asymptotics near the Crack Tip. IUTAM Symposium on Asymptotics, Singularities and Homogenisation in Problems of Mechanics Solid Mechanics and Its Applications. 2004; 113:251-260. DOI:10.1007/1-4020-2604-8_25.

[16] Mishuris G, Öchsner A, Kuhn G. FEM-analysis of nonclassical transmission conditions between elastic structures. Part 2: Stiff imperfect interface. Computers, Materials and Continua. 2006; 4 (3):137-151.

[17] Kuo H-Y. Effective property of multiferroic fibrous composites with imperfect interfaces. Smart Materials and Structures. 2013; 22 (10):105005. DOI:10.1088/0964-1726/22/10/105005. 